\title{
A CONVERSE TO A RESIDUAL FINITENESS THEOREM OF G. BAUMSLAG
}

\author{
M. SHIRVANI
}

(Communicated by Warren J. Wong)

\begin{abstract}
It is shown that if at least two of the factor groups of a nontrivial amalgamated free product $G$ satisfy nontrivial identities, then a special form of the profinite closure of the associated subgroups is necessary (as well as sufficient) for the residual finiteness of $G$. An example shows that the necessity no longer holds if only one of the factor groups satisfies an identity.
\end{abstract}

A group $G$ is said to be residually finite if the intersection of all normal subgroups of finite index in $G$ is trivial. In [1] G. Baumslag gives a sufficient condition for an amalgamated free product of two groups to be residually finite. The condition can be stated for an arbitrary number of factors as follows: let

$$
G=\left\langle * A_{i}: H_{i}=H_{j} \text { via } \theta_{i j} \text { for all } i, j \in \Lambda\right\rangle
$$

be a proper amalgamated free product, so each $H_{i}$ is a proper subgroup of $A_{i}$, and the $\theta_{i j}: H_{i} \rightarrow H_{j}$ are isomorphisms satisfying the consistency conditions $\theta_{i j} \theta_{j k}=$ $\theta_{i k}$ and $\theta_{i j} \theta_{j i}=\theta_{i i}=\operatorname{id}_{H_{i}}$. Let $I$ be the set of all sequences $P=\left(P_{i}\right)_{i \in \Lambda}$ such that $P_{i} \triangleleft_{f} A_{i}$ for all $i,\left(P_{i} \cap H_{i}\right) \theta_{i j}=P_{j} \cap H_{j}$ for all $i, j$, and there exists an integer $m$ such that $\left|A_{i}: H_{i}\right| \leq m$ for all $i$. Given $P \in I$ one can form the amalgamated free product $G_{P}$ of the groups $\bar{A}_{i}=A_{i} / P_{i}$ with amalgamated subgroups $\bar{H}_{i}$ and isomorphisms induced by the $\theta_{i j}$. There is an obvious epimorphism $\pi_{P}: G \rightarrow G_{P}$, and since each $G_{P}$ is residually finite it is easy to see that the following holds.

THEOREM 1. Let $G$ be as in (1). Then $G$ is residually finite if and only if $\bigcap_{P \in I} \operatorname{ker} \pi_{P}=\langle 1\rangle$.

Baumslag's criterion [1, Proposition 3] reads as follows:

Let $G$ as in (1). Assume that

$$
\begin{gathered}
H_{i}=\bigcap_{P \in I} H_{i} P_{i} \quad \text { for all } i \in \Lambda, \\
\bigcap_{P \in I} P_{i}=\langle 1\rangle \quad \text { for all } i \in \Lambda .
\end{gathered}
$$

Then $G$ is residually finite.

This follows from Theorem 1 since (2) and (3) ensure that any nontrivial element of $G$ can be mapped to an element of the same length in some $G_{P}$.

Received by the editors January 15, 1988.

1980 Mathematics Subject Classification (1985 Revision). Primary 20E26; Secondary 20E06.

Key words and phrases. Residual finiteness, amalgamated free product, tree product, nontrivial identity on groups.

This work was supported in part by grants from the NSERC. 
It is trivial that (3) is also necessary for the residual finiteness of $G$, since $\bigcap_{P \in I} P_{i}=A_{i} \cap \bigcap_{P \in I} \operatorname{ker} \pi_{P}$ for all $i$. The results on the residual finiteness of amalgamated free products published so far either utilize (2), or have hypotheses which imply (2). This raises the question of the necessity of this condition. In this direction Gregorac [2] shows that if two of the $A_{i}$ satisfy nontrivial identities and either $\left|A_{i}: H_{i}\right| \geq 3$ or the identities do not hold in the infinite dihedral group, then $(2)$ is also necessary for the residual finiteness of $G$. These restrictions, however, are by no means essential, as the following shows:

THEOREM 2. Let $G$ be as in (1), and assume that at least two of the $A_{i}$ satisfy nontrivial identities. Then conditions (2) and (3) are necessary as well as sufficient for the residual finiteness of $G$.

That some restriction on the $A_{i}$ is necessary is shown by the following example:

EXAMPLE. There exists a residually finite amalgamated free product $G=\langle A * B$; $H=K$ via $\theta\rangle$ where $H \varsubsetneqq A, K \varsubsetneqq B$, the group $A$ is torsion-free abelian, and $H$ is not profinitely closed in $A$.

Proof OF THEOREM 2. We need to recall the following fact [4, Lemma 2]: a group $A$ satisfying a nontrivial identity also satisfies a nontrivial identity of the form

$$
\begin{aligned}
& W=W\left(y, x_{1}, x_{2}\right)=W_{0} y^{\varepsilon_{1}} W_{1} y^{\varepsilon_{2}} \cdots y^{\varepsilon_{n}} W_{n} \\
& \quad \text { where } n \geq 1, \varepsilon_{r}= \pm 1 \text { for all } r \\
& \quad \text { and } W_{0}, \ldots, W_{n} \in\left\{x_{1}^{ \pm 1}, x_{2}^{ \pm 1},\left(x_{1} x_{2}^{-1}\right)^{ \pm 1}\right\}
\end{aligned}
$$

We also write $W(A)=\left\langle W\left(a_{1}, a_{2}, a_{3}\right): a_{i} \in A\right\rangle$. Assume that $G$ is residually finite, and suppose $H_{k} \neq \bigcap_{P \in I} H_{k} P_{k}$ for some $k \in \Lambda$. Throughout, $h$ denotes a fixed element in $\left(\bigcap_{P \in I} H_{k} P_{k}\right) \backslash H_{k}$. By assumption two of the factor groups, $A_{i}$ and $A_{j}$, say, satisfy nontrivial identities. There are two separate cases to be considered.

Case $1 . k \notin\{i, j\}$. We first show that $\left|A_{i}: H_{i}\right|=2=\left|A_{j}: H_{j}\right|$. For assume, to the contrary, that $\left|A_{i}: H_{i}\right| \geq 3$, and let $\left\{1, a_{1}, a_{2}\right\}$ be part of a right transversal of $H_{i}$ in $A_{i}$. We may suppose that $A_{i}$ satisfies an identity $W$ as in (4). Consider the element

$$
g=W\left(h, a_{1}, a_{2}\right)=W_{0}\left(a_{1}, a_{2}\right) h^{\varepsilon_{1}} \cdots h^{\varepsilon_{n}} W_{n}\left(a_{1}, a_{2}\right) .
$$

Clearly $g$ is reduced as written since each $W_{r}\left(a_{1}, a_{2}\right) \in A_{i} \backslash H_{i}$. Hence $g \neq 1$. If $P \in I$ then $h \pi_{P} \in H_{k} \pi_{P}=H_{i} \pi_{P} \subseteq A_{i} \pi_{P}$, whence $g \pi_{P} \in W\left(A_{i}\right) \pi_{P}=\langle 1\rangle$ since $A_{i}$ satisfies $W$. We have therefore found a nontrivial element in $\bigcap_{P \in I} \operatorname{ker} \pi_{P}$, contradicting the residual finiteness of $G$, by Theorem 1 . Since $A_{i} \neq H_{i}$ it follows that we must have $\left|A_{i}: H_{i}\right|=2$. Similarly $\left|A_{j}: H_{j}\right|=2$. From this we obtain another contradiction, showing that Case 1 cannot arise. To this end choose $b_{i} \in$ $A_{i} \backslash H_{i}$ and $b_{j} \in A_{j} \backslash H_{j}$. Now $H_{k}$, being isomorphic to $H_{i} \subseteq A_{i}$, satisfies an identity $W$ of the form (4). Consider the element

$$
g=W\left(h, h^{b_{i}}, h^{b_{j}}\right)=W_{0}\left(h^{b_{i}}, h^{b_{j}}\right) h^{\varepsilon_{1}} \cdots h^{\varepsilon_{n}} W_{n}\left(h^{b_{i}}, h^{b_{j}}\right) .
$$

For $0 \leq s \leq n$ the element $W_{s}\left(h^{b_{i}}, h^{b_{j}}\right)$ is one of $\left(b_{i}^{-1} h b_{i}\right)^{ \pm 1}$ or $\left(b_{j}^{-1} h b_{j}\right)^{ \pm 1}$ or $\left(b_{i}^{-1} h b_{i} b_{j}^{-1} h^{-1} b_{j}\right)^{ \pm 1}$. Since $b_{i}, b_{j}$ and $h$ come from different factors each $W_{s}$ is 
reduced as written and begins and ends with some $b^{ \pm 1}$. Thus $g \neq 1$. Choose any $P \in I$. Then $h \pi_{P} \in H_{k} \pi_{P}$, and since $H_{i}$ is normalized by $b_{i}$ we have

$$
h^{b_{i}} \pi_{P} \in H_{k}^{b_{i}} \pi_{P}=H_{i}^{b_{i}} \pi_{P}=H_{i} \pi_{P}=H_{k} \pi_{P} .
$$

Similarly $h^{b_{j}} \pi_{P} \in H_{k} \pi_{P}$, and consequently $g \pi_{P} \in W\left(H_{k}\right) \pi_{P}=\langle 1\rangle$. This is a contradiction. Case 1, therefore, cannot arise.

Case 2. $k \in\{i, j\}$, say $k=i$. The first part of the proof of Case 1 shows that $\left|A_{j}: H_{j}\right|=2$. Let $\left\{1, a_{1}, a_{2}\right\}$ be part of a right transversal of $H_{k}$ in $A_{k}$. We may assume that $A_{k}\left(=A_{i}\right)$ satisfies an identity $W$ of the form (4). Choose $b \in A_{j} \backslash H_{j}$ (so $b$ normalizes $H_{j}$ ), and let

$$
g=W\left(h^{b}, a_{1}, a_{2}\right)=W_{0}\left(a_{1}, a_{2}\right) b^{-1} h^{\varepsilon_{1}} b \cdots b^{-1} h^{\varepsilon_{n}} b W_{n}\left(a_{1}, a_{2}\right) .
$$

By construction $h^{ \pm 1}$ and the $W_{s}$ come from $A_{k} \backslash H_{k}$, while $b^{ \pm 1} \in A_{j} \backslash H_{j}$. Thus $g \neq 1$. For any $P \in I$ we have

$$
h^{b} \pi_{P} \in H_{k}^{b} \pi_{P}=H_{j}^{b} \pi_{P}=H_{j} \pi_{P}=H_{k} \pi_{P} \subseteq A_{k} \pi_{P}
$$

and consequently $g \pi_{P} \in W\left(A_{k}\right) \pi_{P}=\langle 1\rangle$. This is impossible in view of the residual finiteness of $G$. We have now shown that $H_{k}$ has index 2 in $A_{k}$. Consider the sequence $Q=\left(Q_{\lambda}\right)_{\lambda \in \Lambda}$ where $Q_{k}=H_{k}$ and $Q_{\lambda}=A_{\lambda}$ if $\lambda \neq k$. Clearly $Q \in I$. But then $\bigcap_{P \in I} H_{k} P_{k} \subseteq H_{k} Q_{k}=H_{k}$. This final contradiction proves the result.

We now turn to the example. Let $A=\times_{i=1}^{\infty}\left\langle a_{i}\right\rangle$ be a direct product of infinite cyclic groups, and let $r \geq 2$ be a fixed integer. Consider any set $\left\{h_{i}: i=1,2, \ldots\right\}$ of elements of $A$, and let

$$
G=\left\langle A, x_{i}, i=1,2, \cdots: x_{i}^{r}=h_{i} \text { for } i \geq 1\right\rangle .
$$

Clearly $G$ is a tree product with vertex groups $A_{0}=A$ and $A_{i}=\left\langle x_{i}\right\rangle$, and edge subgroups $H_{i 0}=\left\langle x_{i}^{r}\right\rangle$ and $H_{0 i}=\left\langle h_{i}\right\rangle$. Let $H=\left\langle h_{i}: i \geq 1\right\rangle, K=\left\langle x_{i}^{r}: i \geq 1\right\rangle$ and $B=\left\langle x_{i}: i \geq 1\right\rangle$. By a result of $\mathrm{H}$. Neumann [3, Theorem 6.02] the tree product $G$ can also be presented as the amalgamated free product of $A$ and $B$, amalgamating the proper subgroups $H$ and $K$. We claim that $G$ is residually finite. Granted that this is so, we can set $h_{1}=a_{1}^{2}$ and $h_{i}=a_{i+1}^{2} a_{i}^{-1}$ for $i \geq 2$ to find that $H$ is not profinitely closed in $A$, since $A / H \cong C_{2}$ (the Prüfer 2-group).

It remains to show that $G$ is residually finite. To this end it is enough to verify the following:

(a) Each $H_{i-}$ is profinitely closed in $A_{i}$, for $i \geq 0$;

(b) Given any $M \triangleleft_{f} A_{j}(j \geq 0)$ there exists a sequence $\left(Q_{i}\right)_{i \geq 0}$ such that $Q_{i} \triangleleft_{f} A_{i},\left|A_{i}: Q_{i}\right|$ is bounded for all $i, Q_{0} \cap\left\langle h_{i}\right\rangle=Q_{i} \cap\left\langle x_{i}^{r}\right\rangle$ for all $i \geq 1$, and $Q_{j}=M$.

The residual finiteness of $G$ will then follow from [5]. It is well known that condition (a) holds, so we turn to (b).

Suppose $M \triangleleft_{f} A$ is given. Set $\left\langle h_{i}^{m_{i}}\right\rangle=M \cap\left\langle h_{i}\right\rangle$ for $i \geq 1$. Clearly $m_{i} \leq|A: M|$ for all $i$, and so the sequence $\left(P_{i}\right)_{i \geq 0}$, where $P_{0}=M$ and $P_{i}=\left\langle x_{i}^{r m_{i}}\right\rangle$, satisfies (b). Now suppose $L \triangleleft_{f} A_{i}$, where $i \geq 1$, is given, and set $L \cap H_{i 0}=\left\langle x_{i}^{r s}\right\rangle$, where $s$ depends on $L$. Since the factor group $A /\left\langle h_{i}^{s}\right\rangle$ is residually finite and $\left\langle h_{i}\right\rangle /\left\langle h_{i}^{s}\right\rangle$ is finite, there exists $M \triangleleft_{f} A$ with $M \cap\left\langle h_{i}\right\rangle=\left\langle h_{i}^{s}\right\rangle$. By the earlier part of this paragraph we can find a sequence $\left(P_{i}\right)$ satisfying (b) such that $P_{0}=M$. The sequence $Q$ obtained from $P$ by replacing $P_{i}$ by $L$ also satisfies (b), and $Q_{i}=L$. Thus condition (b) also holds, as claimed. 


\section{REFERENCES}

1. G. Baumslag, On the residual finiteness of generalized free products of nilpotent groups, Trans. Amer. Math. Soc. 106 (1963), 193-209.

2. R. J. Gregorac, On residually finite generalized free products, Proc. Amer. Math. Soc. 24 (1970), 553-555.

3. H. Neumann, Generalized free products with amalgamated subgroups. I, Amer. J. Math. 70 (1948), 590-625.

4. M. Shirvani, On residually finite $H N N$-extensions, Arch. Math. 44 (1985), 110-115.

5. __ On residually finite graph products, J. Pure Appl. Algebra 49 (1987), 281-282.

Department of Mathematics, University of Alberta, Edmonton, Alberta, CANADA T6G 2G1 